\title{
Usefulness of Optical Coherence Tomography in the Preoperative Assessment of Nail Deformities
}

\author{
Han Gyu Cha ${ }^{1}$, Eun Soo Park ${ }^{2}$, \\ Seung Min Nam ${ }^{2}$ \\ ${ }^{1}$ Department of Plastic and \\ Reconstructive Surgery, Asan Medical \\ Center, University of Ulsan College of \\ Medicine, Seoul; ${ }^{2}$ Department of Plastic \\ and Reconstructive Surgery, \\ Soonchunhyang Bucheon Hospital, \\ Soonchunhyang University College of \\ Medicine, Bucheon, Korea
}

This work was supported by the Soonchunhyang University Research Fund.

No potential conflict of interest relevant to this article was reported.

\begin{abstract}
Secondary nail deformities are often seen at some time after fingertip and nail bed injuries. A proper evaluation of the fingernail enables appropriate diagnosis and treatment, especially in patients who need surgical management. To date, ultrasound and magnetic resonance imaging are the only diagnostic tools that most surgeons use in cases of nail deformities. Herein, we report the case of a patient with a nail deformity who underwent successful correction based on the preoperative use of real-time optical coherence tomography.
\end{abstract}

Keywords Nails malformed, Reconstructive surgical procedures, Tomography optical coherence

\section{INTRODUCTION}

The most common injuries of the hand are fingertip and nail bed injuries, and secondary nail deformities are often seen at some time after the injury. To date, ultrasound and magnetic resonance imaging (MRI) are the only diagnostic tools that most surgeons use in cases of nail deformities, besides the naked eye. Optical coherence tomography (OCT) is a non-invasive, non-destructive, real-time imaging technique for the morphological investigation of tissue. OCT can image structures up to $3 \mathrm{~mm}$ in depth in highly scattering tissue, and it is thus ideal for assessing deformities of the nail. Herein, we introduce OCT as a useful preoperative assessment tool in cases of fingernail deformities to detect morphological changes of the nail bed.

Received: May 28, 2018 Revised: Jul 12, 2018 Accepted: Jul 16, 2018 Correspondence: Eun Soo Park Department of Plastic and Reconstructive Surgery, Soonchunhyang Bucheon Hospital, Soonchunhyang University College of Medicine, 170 Jomaru-ro, Wonmi-gu, Bucheon 14584, Korea. E-mail: peunsoo@schmc.ac.kr

Copyright @ 2018 The Korean Society for Aesthetic Plastic Surgery.

This is an Open Access article distributed under the terms of the Creative Commons Attribution Non-Commercial License (http://creativecommons.org/licenses/by-nc/4.0/) which permits unrestricted non-commercial use, distribution, and reproduction in any medium, provided the original work is properly cited. $\quad$ www.e-aaps.org

\section{CASE REPORT}

An 8-year-old girl with a fingernail deformity on her right fifth finger visited our clinic. She had a history of trauma involving her fingertip that occurred 2 years previously. A physical examination revealed a vertically thickened deformity at the central portion of the nail (Fig. 1). OCT was used to detect the invisible deformity under the nail plate. Imaging was performed with a hand-held probe (5 $\mathrm{mW}$ optical power, $855 \mathrm{~nm}$ light source), which was connected to the OCT device (OCTskin; WonTech Co., Ltd., Daejeon, Korea). The OCT device had a spectral bandwidth of $10 \mathrm{~nm}$, yielding a typical imaging depth of $1 \mathrm{~mm}$, with $7.5-\mu \mathrm{m}$ lateral resolution and $10-\mu \mathrm{m}$ axial resolution. The OCT images revealed vertical ridging in the central portion of the nail bed, compared to the normal side (Fig. 2 and 3). As injury of the sterile matrix and germinal matrix was suspected, surgical exploration was performed. Given careful consideration of the direction of the ridge as shown on OCT, the entire nail plate was precisely removed from the underlying nail bed and matrix. The longitudinal deformity of the nail bed was closely correlated with the OCT images (Fig. 4). A scar on the germinal matrix, caused by the previous injury, was also confirmed. The irregularities of the germinal and sterile matrix were trimmed into a straight line and closed accurately with a 7-0 absorbable suture, and a silicone sheet was placed onto the nail fold between the dorsal and ventral floor to prevent scarring. No complications were 


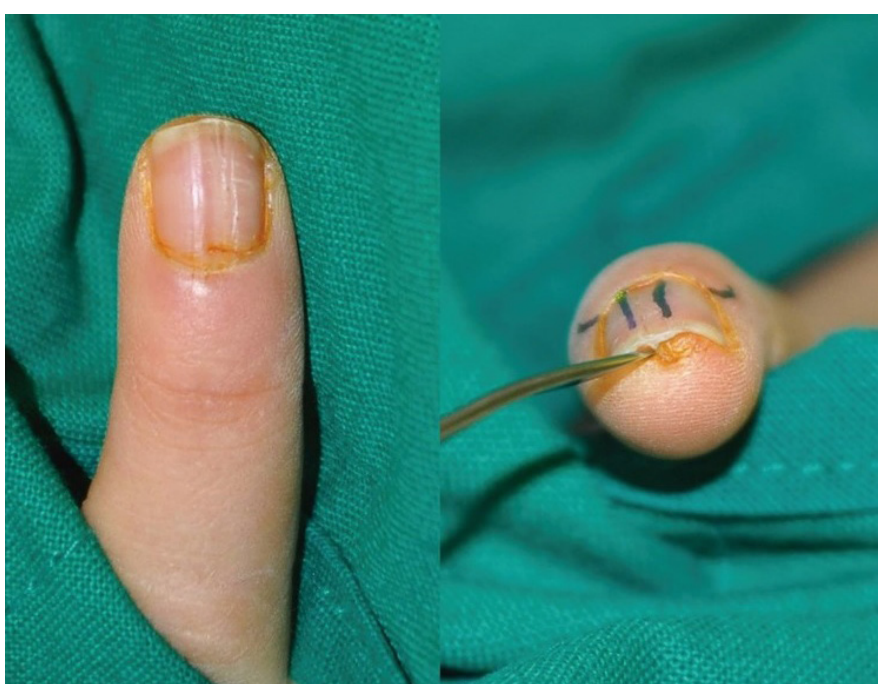

Fig. 1. Preoperative photographic findings of an 8-year-old patient with a longitudinal ridge on her right little finger.
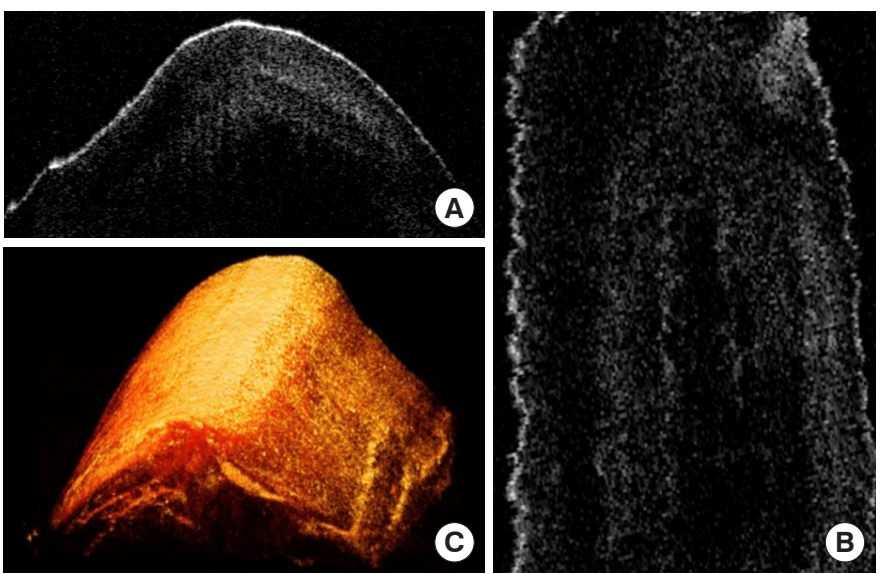

Fig. 2. Optical coherence tomography images of the right little finger with nail deformity. (A) Axial view. (B) Coronal view. (C) The 3-dimensional reconstruction view.

observed over 12 months of follow-up, and when the new nail grew in, it showed a remarkably improved shape (Fig. 5).

\section{DISCUSSION}

Correcting a nail ridge requires excision of the responsible scar or irregular bone edge to form a flat and smooth nail bed surface [1]. To date, most surgeons have predicted the cause of the deformity and performed surgical exploration using the naked eye. MRI has been used as a preoperative assessment tool to image nail disease, but its ability to visualize the nail is limited due to the nail's low water content [2]. Ultrasound is the most widely used imaging tool for nail disease, but it has limitations in that it cannot measure and visualize nail structures on the scale of millimeters $[3,4]$. Further-


Fig. 3. Optical coherence tomography images of the normal left side. (A) Axial view. (B) Coronal view. (C) The 3-dimensional reconstruction view.

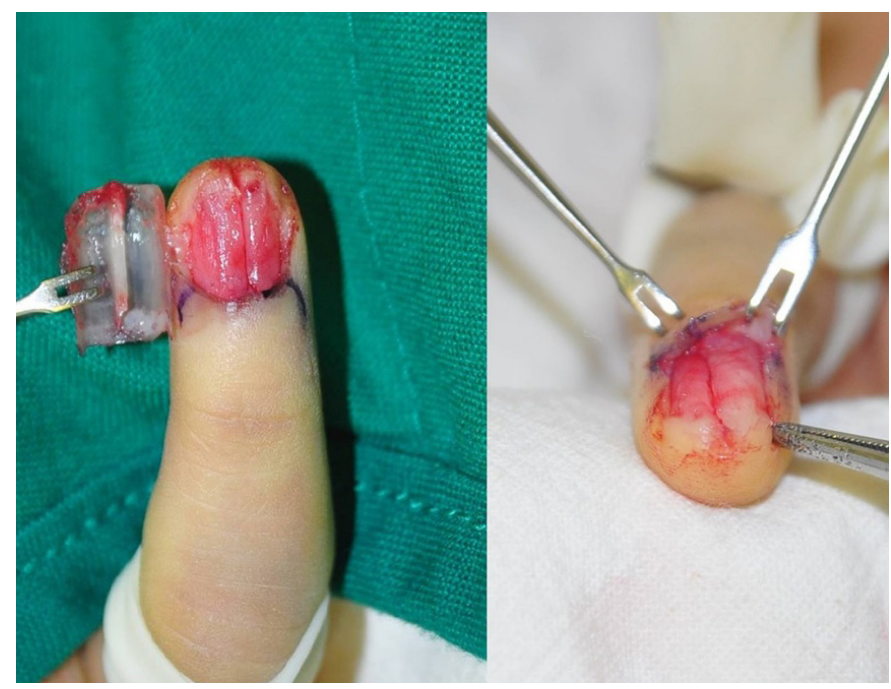

Fig. 4. Intraoperative photographic findings of the nail bed, which were closely correlated with the optical coherence tomography images.

more, both MRI and ultrasound require additional time for the patient to make another appointment with the radiologist and for the interpretation of the resulting images. This is a waste of time for both the patient and the surgeon.

OCT, a non-invasive diagnostic tool, was first introduced to image the human eye in 1988 and was first applied to skin imaging in 1997 [5]. OCT has since been applied to the diagnosis of a wide variety of skin structures and skin cancers in clinical settings [6]. OCT has a resolution of less than a few tens of micrometers, which makes it especially useful for diagnosing skin diseases and deformities in dermatology and plastic surgery. Analogous to ultrasound, OCT uses infrared light and employs interferometric methods to measure optical echo delays and to detect light reflected from with- 


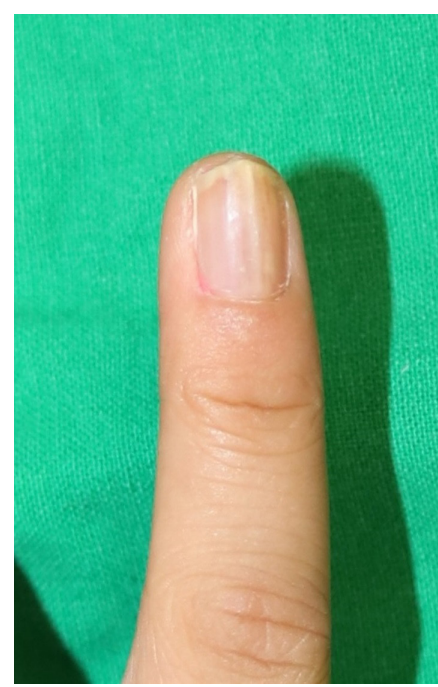

Fig. 5. Photographic findings of the newly grown nail 12 months after surgery.

in the tissue [7]. OCT images are formed by dividing light from an optical source into 2 pathways. The light reflected from the tissue is recombined with light from the reference mirror and detected, forming an interference signal only when the lengths of the tissue and reference paths are matched to within a short coherence length at about $15 \mathrm{~mm}$ from the light source [8]. OCT can provide 3-dimensional reconstruction images that show layers of structures at a constant depth from the surface of the skin and deliver highly accurate information about the target. With OCT, which is conceptually similar to ultrasound, image acquisition can be performed in real-time without requiring ionized radiation. In addition, OCT distinguishes subtle differences between the soft tissues that other devices cannot.

We utilized OCT to identify the accurate shape of a scar beneath the nail, and we directly approached the germinal matrix beneath the eponychium to revise the nailbed. Without help from the OCT, we might have neglected to revise the nailbed around the germinal matrix, potentially resulting in recurrence of the ridge. This result indicates that OCT can be useful for surgeons, as it is a non-invasive and accurate diagnostic tool with minimal side effects. Based on the accurate information provided by OCT, a surgeon can plan a surgical procedure more exactly and correct the defect more precisely, while minimizing the injury to the surrounding nail structures. Above all, the patient can obtain a satisfying result without additional time and expense.

\section{PATIENT CONSENT}

Patient's guardians provided written consent for the use of her images.

\section{REFERENCES}

1. Ashbell TS, Kleinert HE, Putcha SM, et al. The deformed finger nail, a frequent result of failure to repair nail bed injuries. J Trauma 1967;7:17790.

2. Tan AL, Benjamin M, Toumi H, et al. The relationship between the extensor tendon enthesis and the nail in distal interphalangeal joint disease in psoriatic arthritis--a high-resolution MRI and histological study. Rheumatology (Oxford) 2007;46:253-6.

3. Wollina U, Berger M, Karte K. Calculation of nail plate and nail matrix parameters by $20 \mathrm{MHz}$ ultrasound in healthy volunteers and patients with skin disease. Skin Res Technol 2001;7:60-4.

4. Aydin SZ, Castillo-Gallego C, Ash ZR, et al. Ultrasonographic assessment of nail in psoriatic disease shows a link between onychopathy and distal interphalangeal joint extensor tendon enthesopathy. Dermatology 2012;225:231-5.

5. Welzel J, Lankenau E, Birngruber R, et al. Optical coherence tomography of the human skin. J Am Acad Dermatol 1997;37:958-63.

6. Mogensen M, Joergensen TM, Nürnberg BM, et al. Assessment of optical coherence tomography imaging in the diagnosis of non-melanoma skin cancer and benign lesions versus normal skin: observer-blinded evaluation by dermatologists and pathologists. Dermatol Surg 2009; 35:965-72.

7. Park ES. Skin-layer analysis using optical coherence tomography (OCT). Med Laser 2014;3:1-4.

8. Pierce MC, Strasswimmer J, Park BH, et al. Advances in optical coherence tomography imaging for dermatology. J Invest Dermatol 2004; 123:458-63. 